\title{
VACINA CONTRA PAPILOMAVÍRUS HUMANO NA PREVENÇÃO DO CÂNCER CERVICAL
}

\author{
Aíla Marôpo ARÁUJO \\ Cilene Nunes DANTAS ${ }^{2}$ \\ Ana Elza Oliveira de MENDONÇA ${ }^{3}$ \\ Rejane Maria Paiva de MENEZES ${ }^{4}$ \\ Ingrid Gurgel AMORIM ${ }^{5}$ \\ Vinicius Lino de SOUZA NETO ${ }^{6}$
}

\begin{abstract}
${ }^{1}$ Enfermeira. Coordenadora da Vigilância Epidemiológica de Natal/RN. Professora Substituta da Escola de Enfermagem de Natal/UFRN. Membro do grupo de pesquisa Práticas Assistenciais e Epidemiológicas em Saúde e Enfermagem (UFRN/RN/BRASIL). E-mail: ailamaropo@yahoo.com.br

${ }^{2}$ Enfermeira. Mestre em Enfermagem pela UFRN/RN/BRASIL. Enfermeira da Secretaria Municipal de Saúde de Natal. Docente do Curso de Enfermagem da UNIFACEX/RN/BRASIL. E-mail: cilenenunesdantas@bol.com.br

${ }^{3}$ Enfermeira. Doutoranda em Ciências da Saúde pela Universidade Federal do Rio Grande do Norte/UFRN/RN/BRASIL. Professora Mestre da Graduação em Enfermagem da Universidade Faculdade de Ciências, Cultura e Extensão do RN (UNIFACEX). E-mail: a.elza@uol.com.br
\end{abstract}

${ }^{4}$ Enfermeira. Doutora em Enfermagem pela Universidade de São Paulo/USP/SP/BRASIL. Professora do Programa de Pós-Graduação em Enfermagem e do Departamento de Enfermagem da Universidade Federal do Rio Grande do Norte/UFRN/RN/BRASIL. Vice-Coordenadora da Base de Pesquisa Práticas Assistenciais e Epidemiológicas em Saúde e Enfermagem, do Departamento de enfermagem (UFRN/RN/BRASIL). E-mail: rejemene@terra.com.br

5 Enfermeira. Aluna especial de Mestrado do Programa de Pós-Graduação em Enfermagem - Mestrado Acadêmico, da Universidade Federal do Rio Grande do Norte/PPGENF/UFRN. Natal-RN, Brasil. E-mail: ingridgurgel88@gmail.com

${ }^{6}$ Discente do Curso Bacharelado em Enfermagem da Universidade Federal de Campina Grande e Bolsita do Programa de Extensão - PROBEX / Vigência - 2013 - UFCG; Líder do Grupo de pesquisa em Epidemiologia e Saúde Coletiva - GPESC. E-mail: vinolino@hotmail.com

Autor(a) responsável pela troca de correspondência

Aíla Marôpo Araújo

Rua Alziro Zaruh, 22A, Planalto

CEP: 59073-072 - Natal (RN), Brasil

Recebido em: 11/09/2013 - Aprovado em: 13/12/2013 - Disponibilizado em: 15/01/2014

RESUMO: O Papilomavírus Humano é capaz de produzir lesões de pele e mucosas, sendo responsável pelo desenvolvimento de neoplasia intraepitelial e câncer cervical, configurando-se como um problema de saúde pública mundial. A vacina contra papilomavírus pretende reduzir a incidência de infecções pelo papilomavírus humano, principal agente no desenvolvimento de lesões precursoras do câncer cervical. Objetivou-se destacar através do conhecimento científico a relevância da vacina contra papilomavírus humano na prevenção do câncer cervical. Trata-se de uma revisão integrativa da literatura, processada nas bases de dados LILACS, SciELO e MEDLINE, em que foram selecionados 16 artigos, publicados entre 2006 e 2011. Os resultados revelam redução dos índices de infecção por papilomavírus humano e do desenvolvimento da neoplasia cervical em mulheres que utilizaram a vacina. Conclui-se que há necessidade de novos estudos que reforcem a vacinação, como importante medida profilática, apesar dos entraves sociais, econômicos e culturais que concernem à prevenção do câncer cervical. 
Descritores: Infecções por Papilomavírus. Vacinas contra Papilomavírus Humano. Neoplasias do Colo do Útero. Prevenção de Câncer de Colo Uterino. Atenção Primária a Saúde.

\title{
HUMAN PAPILOMAVIRUS VACCINE IN CER VICAL CANCER PREVENTION
}

\begin{abstract}
The Human Papilomavirus is capable of producing skin lesions and mucous membranes, being responsible for the development of intraepithelial neoplasia and cervical cancer, becoming a worldwide public health problem. The papilomavirus vaccine aims to reduce the incidence of infection by the human papilomavirus, the main agent in developing precursor lesions and cervical cancer. This study's objective highlights the relevance of human papilomavirus vaccine in cervical cancer prevention. This is an integrative literature review, processed in the LILACS, SciELO and MEDLINE databases, in which 16 articles published between 2006 and 2011 were selected. The results show there is a reduction in Human Papilomavirus infections and the development Cervical Neoplasms in women who used the vaccine. However, there is need for further studies which reinforce vaccination is an important prophylactic measure, despite the social, economic and cultural obstacles that concern the prevention of cervical cancer.

Descriptors: Papillomavirus Infections. Papillomavirus Vaccines. Uterine Cervical Neoplasms. Cervix Neoplasms Prevention. The Primary Health.
\end{abstract}

\section{INTRODUÇÃO}

O câncer tem impacto global sobre a população e o número de casos praticamente dobrou em 30 anos, segundo estimativas recentes do relatório da Agência Internacional para Pesquisa em Câncer (IARC) da Organização Mundial de Saúde (OMS). Assim, foi previsto que ocorreriam cerca de 12 milhões de novos casos de câncer e 7 milhões de óbitos em 2008. A explicação para o percentual elevado de óbitos por câncer está diretamente relacionada à maior exposição dos indivíduos a fatores de risco cancerígenos ${ }^{(1)}$.

$\mathrm{Na}$ população feminina os tipos de câncer que se mostraram mais frequentes, respectivamente, foram de mama, colo do útero, cólon e reto, estômago e pulmão. $\mathrm{O}$ câncer de colo do útero, ou câncer cervical, é o segundo tipo de câncer mais incidente entre as mulheres, e constitui-se um problema de saúde pública mundial, em especial nos países em desenvolvimento, onde ocorrem $80 \%$ dos casos. Sabe-se que o Papilomavírus Humano (HPV) está diretamente relacionado ao desenvolvimento da neoplasia intraepitelial (NIC) de alto grau e do câncer invasivo, embora não seja causa suficiente ${ }^{(2)}$.

Do ponto de vista histopatológico, as neoplasias intraepiteliais cervicais classificam-se em lesões reversíveis, desde que devidamente reconhecidas e tratadas. São elas: a NIC I (lesão intraepitelial de baixo grau, com anormalidades do epitélio em um terço proximal da membrana), a NIC II (lesão intraepitelial de alto grau, que compromete até dois terços da espessura do epitélio), a NIC III (lesão intraepitelial de alto grau, cujas alterações compreendem quase toda a espessura do 
epitélio, poupando as células mais superficiais) e o carcinoma in situ ${ }^{(2)}$.

Por sua vez, a infecção pelo HPV é tida como principal fator de risco para o desenvolvimento das lesões precursoras do câncer cervical, classificado atualmente na família Papillomaviridae. Há mais de 100 tipos de vírus descritos, dos quais cerca de 40 são sexualmente transmissíveis e provocam as doenças mais frequentes na atualidade. Os vírus do tipo 16, 18, 31, 33, $35,39,45,51,52,56,58,59$ e 68 são considerados de alto risco para o desenvolvimento da neoplasia do colo do útero e seus precursores, as neoplasias intraepiteliais cervicais ${ }^{(3-4)}$.

A associação entre o HPV e o câncer foi comprovada em 1995 através de uma pesquisa realizada pela IARC, que conduziu um estudo multicêntrico de casos e controles. Foram utilizados dois mil casos de câncer cervical e dois mil controles, sendo observada uma forte associação entre esse tipo de câncer e a presença de qualquer tipo de HPV, de forma que há o risco dessa associação ser superior a 100, comprovando-se que nenhum outro fator de risco para a neoplasia cervical tem magnitude comparável. Nessa perspectiva, entende-se que o investimento na prevenção e no diagnóstico precoce possibilita maiores benefícios sociais e econômicos do que o custeio do tratamento da patologia em fase avançada $^{(2-3)}$.

A infecção pelo HPV configura-se, portanto, como problema de saúde pública mundial, e no âmbito da prevenção do câncer cervical as vacinas contra HPV representam um método profilático eficaz na redução da infecção e da consequente instalação da lesão cervical ou desenvolvimento da neoplasia. Assim, é necessário o conhecimento sobre suas formas de transmissão, diagnóstico, tratamento e prevenção das infecções ocasionadas pelo $\mathrm{HPV}^{(2-3)}$.

Pesquisar o conhecimento acerca do HPV, como fator de risco para o desenvolvimento de câncer de colo uterino, é de suma relevância para a redução da morbidade e mortalidade na saúde da mulher, pois sabe-se que a prevenção das doenças transmissíveis, como a infecção por HPV, representa um enorme desafio não apenas para os gestores de saúde, mas para todos os profissionais dessa área. Ao destacar a importância da vacina contra o Papilomavírus Humano no âmbito da prevenção do câncer do colo do útero, pretende-se responder ao seguinte questionamento: Qual a contribuição da vacina contra HPV para a prevenção do câncer cervical?

Portanto, o propósito deste estudo é destacar através do conhecimento científico a relevância da vacina contra 
papilomavírus humano na prevenção do câncer cervical.

\section{MÉTODOS}

Trata-se de uma revisão integrativa da literatura, que tem como finalidade reunir e sintetizar resultados de estudos já investigados sobre a vacina contra papilomavírus humano na prevenção do câncer cervical, de maneira sistemática e ordenada, de forma a contribuir para o conhecimento do tema investigado ${ }^{(5)}$. Essa abordagem metodológica tem uma compreensão abrangente de determinado fenômeno ou problema de saúde, além de possibilitar diferentes perspectivas acerca do fenômeno em estudo ${ }^{(6)}$.

Este estudo de revisão integrativa percorreu seis etapas. A primeira consistiu na identificação do problema e na seleção da questão de pesquisa que orientou a elaboração da revisão bibliográfica ${ }^{(5,7)}$.

A busca dos artigos foi realizada nas bases de dados: Sistema Latino-Americano e do Caribe de Informação em Ciências da Saúde (LILACS), Scientific Electronic Library Online (SciELO) e Medical Literature Analysisand Retrieval System Online (MEDLINE), durante o mês de abril de 2012. Utilizaram-se como descritores controlados: "vacinas contra papillomavírus humano", "neoplasias do colo do útero" e "prevenção de câncer de colo uterino", conforme a Biblioteca Virtual em Saúde (BIREME) e os Descritores em Ciências da Saúde (DeCS). $\mathrm{Na}$ segunda etapa, se determinou os critérios de inclusão e exclusão. Como critérios de inclusão, definiram-se: publicações em formato de texto completo, no período entre 2006 e 2011, nos idiomas em português, inglês e espanhol. Por sua vez, os critérios de exclusão referiram-se às publicações não condizentes com a questão de pesquisa, que tivessem duplicidades entre as bases de dados consultadas, e não apresentaram em seu título a correlação entre vacina contra HPV e câncer cervical.

A busca totalizou 1.306 publicações. Destas, 1.303 referem-se aos seguintes descritores correlacionados: 1-“vacinas contra papillomavírus humano" e "neoplasias do colo do útero"; 2"prevenção de câncer de colo uterino" e "vacinas contra papilomavírus humano"; 3-“vacina contra papilomavírus humano" e "neoplasias do colo do útero" e 4"prevenção de câncer de colo uterino". Assim, obteve-se com a correlação desses descritores, na base de dados Lilacs: 28; na Scielo: uma e, na Medline: 1.277. Foram excluídas 1.288 publicações decorrentes de repetição entre as bases citadas, por não atenderem aos critérios estabelecidos. As bases de dados com maior quantitativo de publicações foram Medline (9) e Lilacs (7). 
Portanto, a amostra total resultou em 16 publicações.

$\mathrm{Na}$ sequência, foi realizada uma leitura e interpretação dos estudos selecionados, com a identificação dos conteúdos e ideias centrais em comum, ou não, relacionando os diferentes aspectos abordados sobre o tema entre os estudos, assim como, os conteúdos conflitantes aos objetivos propostos (Tabela 1).

A próxima etapa oportunizou a síntese dos principais resultados, fazendose uma análise comparativa entre o conhecimento teórico presente nos estudos e as conclusões apresentadas. Para finalizar, obteve-se uma síntese do conhecimento analisado, a partir da qual foram apresentados os principais resultados e conclusões.

\section{RESULTADOS E DISCUSSÃO}

\section{A Tabela 1 indica a distribuição dos} artigos conforme o local de indexação, título do artigo, periódico, ano de publicação, idioma, tipo de estudo e consideração sobre a temática.

Tabela 1- Identificação dos estudos incluídos na revisão literária, 2012. (N-16)

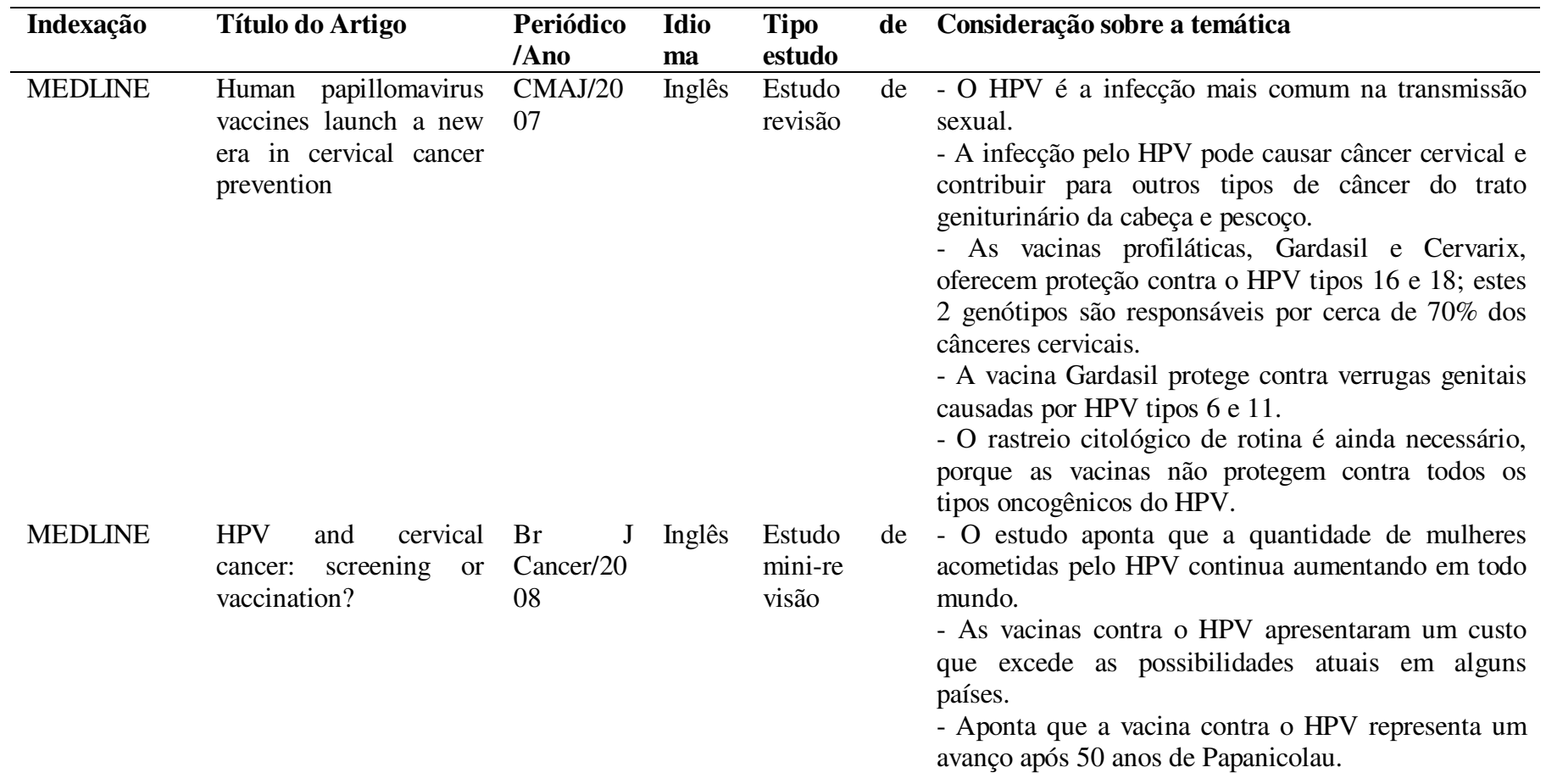




\begin{tabular}{|c|c|c|c|c|c|}
\hline Indexação & Título do Artigo & $\begin{array}{l}\text { Periódico } \\
\text { /Ano }\end{array}$ & $\begin{array}{l}\text { Idio } \\
\text { ma }\end{array}$ & $\begin{array}{l}\text { Tipo } \\
\text { estudo }\end{array}$ & Consideração sobre a temática \\
\hline MEDLINE & $\begin{array}{l}\text { Knowledge of cervical } \\
\text { cancer prevention and } \\
\text { human papillomavirus } \\
\text { among women with } \\
\text { HIV }\end{array}$ & $\begin{array}{l}\text { Gynecol } \\
\text { Oncol/201 } \\
0\end{array}$ & Inglês & $\begin{array}{l}\text { Estudo } \\
\text { Coorte }\end{array}$ & $\begin{array}{l}\text { - O estudo avaliou que as mulheres HIV positivas } \\
\text { compreendiam melhor acerca da prevenção do } \\
\text { câncer cervical, do que as mulheres HIV negativas. } \\
\text { - Cerca de metade das mulheres com HIV positivo } \\
\text { consideram a vacina contra o HPV como importante } \\
\text { na prevenção do câncer cervical. }\end{array}$ \\
\hline MEDLINE & $\begin{array}{l}\text { Multi-site study of } \\
\text { HPV type-specific } \\
\text { prevalence in women } \\
\text { with cervical cancer, } \\
\text { intraepithelial neoplasia } \\
\text { and normal cytology, in } \\
\text { England }\end{array}$ & $\begin{array}{l}\mathrm{Br} \quad \mathrm{J} \\
\text { Cancer/20 } \\
10\end{array}$ & Inglês & $\begin{array}{l}\text { Estudo de } \\
\text { coorte } \\
\text { transversal }\end{array}$ & $\begin{array}{l}\text { - Os estudos internacionais apontam para a } \\
\text { associação entre o HPV e o câncer cervical. } \\
\text { - O HPV } 16 \text { foi o tipo mais encontrado em todos os } \\
\text { graus do câncer do colo uterino. } \\
\text { - Destaca-se que a implantação do programa de } \\
\text { imunização cervical na Inglaterra com impacto } \\
\text { positivo. }\end{array}$ \\
\hline MEDLINE & $\begin{array}{l}\text { Estimating the long- } \\
\text { term impact of a } \\
\text { prophylactic human } \\
\text { papillomavirus } 16 / 18 \\
\text { vaccine on the burden } \\
\text { of cervical cancer in the } \\
\text { UK }\end{array}$ & $\begin{array}{l}\mathrm{Br} \quad \mathrm{J} \\
\text { Cancer/20 } \\
07\end{array}$ & Inglês & $\begin{array}{l}\text { Ensaio } \\
\text { clínico }\end{array}$ & $\begin{array}{l}\text { - O estudo aponta o modelo de processo de Markov, } \\
\text { desenvolvido para avaliar a história do tipo } \\
\text { específico de infecção por HPV e a progressão das } \\
\text { lesões cervicais. } \\
\text { - O estudo aponta o impacto da vacinação contra o } \\
\text { HPV 16/18 na saúde pública. }\end{array}$ \\
\hline MEDLINE & 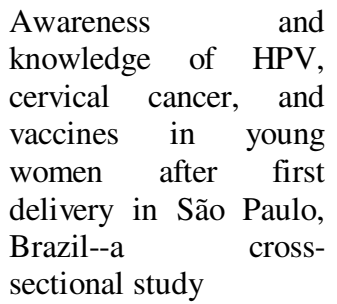 & $\begin{array}{l}\text { BMC } \\
\text { Womens } \\
\text { Health/20 } \\
10\end{array}$ & Inglês & $\begin{array}{l}\text { Estudo } \\
\text { Transversal }\end{array}$ & $\begin{array}{l}\text { - Enfatiza a associação entre o HPV e o câncer } \\
\text { cervical. } \\
\text { - Destaca para a importância da informação entre as } \\
\text { adolescentes do estudo acerca do HPV e seus } \\
\text { desdobramentos. } \\
\text { - Propõe intervenções educativas para incentivar } \\
\text { primíparas e secundigestas na prevenção do câncer } \\
\text { cervical. }\end{array}$ \\
\hline
\end{tabular}




\begin{tabular}{|c|c|c|c|c|c|}
\hline Indexação & Título do Artigo & $\begin{array}{l}\text { Periódico } \\
\text { /Ano }\end{array}$ & $\begin{array}{l}\text { Idio } \\
\text { ma }\end{array}$ & $\begin{array}{l}\text { Tipo } \\
\text { estudo }\end{array}$ & Consideração sobre a temática \\
\hline LILACS & $\begin{array}{l}\text { Que hay en el horizonte } \\
\text { sobre el virus del } \\
\text { papiloma humano, } \\
\text { vacunas y el control del } \\
\text { cáncer cervical }\end{array}$ & $\begin{array}{l}\text { Rev. peru. } \\
\text { med. exp. } \\
\text { salud } \\
\text { publica/20 } \\
07\end{array}$ & $\begin{array}{l}\text { Espan } \\
\text { hol }\end{array}$ & $\begin{array}{l}\text { Estudo de } \\
\text { revisão }\end{array}$ & $\begin{array}{l}\text { - O estudo aponta os diferentes aspectos } \\
\text { epidemiológicos, acerca do câncer cervical por HPV } \\
\text { e das vacinas contra o HPV. } \\
\text { - Destaca que os países em desenvolvimento } \\
\text { precisam de meios simples e baratos para adquirirem } \\
\text { vacina, bem como sua incorporação no setor público. }\end{array}$ \\
\hline
\end{tabular}

Após as leituras das publicações, foram elencados os dois principais eixos temáticos surgidos: $\mathrm{O}$ papilomavírus humano e repercussões no câncer cervical; e Câncer cervical e a vacina contra HPV; os quais acompanham essa organização na apresentação dos resultados a seguir.

\section{O papilomavírus humano e as repercussões no câncer cervical}

A família de vírus HPV é composta por mais de 100 diferentes genótipos virais, sendo os tipos 16 e 18 identificados em cerca de $70 \%$ dos casos de câncer cervical, enquanto que os tipos 6 e 11 podem causar verrugas genitais ${ }^{(8)}$. O HPV é responsável por cerca de 500.000 casos de câncer de colo uterino anualmente, e 10 milhões de novos casos de lesões que antecedem o câncer cervical de graus II ou III, precursores imediatos para malignidade (9)

Estudo ${ }^{(10)}$ coloca que o HPV tem papel central na carcinogênese cervical das

lesões do colo do útero, bem como uma grande percentagem de outros tumores anogenitais e tumores de orofaringe.

Mediante os dados supracitados, observa-se que $100 \%$ dos artigos encontrados apontam de fato para uma relação existente entre o HPV e o câncer cervical, e destacam, entre os principais fatores implicados no processo de infecção, a presença de doença sexualmente transmissível anterior, história pessoal e familiar de HPV ou câncer cervical, idade, conhecimento acerca da doença, de modo que apresentam relação entre o HPV e o câncer cervical.

Outros estudos colocam que as relações entre a infecção pelo HPV, o NIC e o carcinoma invasor são bem estabelecidas, tanto que o HPV foi identificado em $99 \%$ dos casos de carcinomas cervicais, embora não seja a causa suficiente. De fato, infecções persistentes determinadas pelos HPVs oncogênicos aumentam o risco da 
neoplasia intraepitelial e do câncer (3-4,1112).

O câncer cervical configura-se como a segunda neoplasia maligna mais frequente para a população feminina, perdendo apenas para o câncer de pele. Dessa forma, trata-se de um problema de saúde pública. A cada ano tem-se diagnosticado 500.000 novos casos e 230.000 óbitos devido a essa neoplasia ${ }^{(1,}$ 11-13)

Mundialmente, são estimados 32 milhões de casos novos de verrugas genitais a cada ano, sendo a grande maioria associada aos HPVs6 (70\% dos casos) e 11 (20\% dos casos). Estima-se que 5,5 milhões de pessoas tornam-se infectadas pelo HPV a cada ano nos EUA, e o sexo feminino é o mais acometido ${ }^{(10,14)}$.

Aproximadamente $40 \%$ das mulheres sexualmente ativas são infectadas pelo HPV. No Brasil, o HPV16 é o tipo predominante nos cânceres cervicais invasivos nas Regiões Sul, Centro-Oeste, Nordeste, Norte e Sudeste, com prevalências de 52\%, 57\%, 59\%, 43,5\% e $52 \%$, respectivamente. Em relação a outros tipos de HPV (18, 31 e 33), observam-se variações regionais, sendo que na maioria das regiões o segundo mais prevalente é o HPV18, com exceção da Região CentroOeste, em que predomina o HPV33, e na Região Nordeste, onde o HPV31 é o segundo em prevalência ${ }^{(3)}$.
Estudos colocam a possibilidade de a população feminina, em sua maioria, adquirir a infecção pelo HPV em algum momento de sua vida. Também indicam que a maioria das infecções poderia ser resolvida de forma espontânea, o fato, porém, é que a incidência de infecção pelo HPV é elevada entre as mulheres sexualmente ativas com até 25 anos de idade $^{(4,10-11)}$.

Os responsáveis pela maioria das lesões por HPV induzidas na região anogenital frequentemente são os HPVs 6, 11, 16 e 18. Os tipos diferentes desse vírus encontram-se reunidos em dois grupos, a saber: o de baixo e o de alto risco oncogênico. Os tipos de HPVs de baixo risco mais frequentes são $6,11,40,42,43$, $44,54,61,70,72$ e 81 , e os de alto risco são $16,18,31,33,35,39,45,51,52,56$, $58,66,73$ e $82^{(12)}$.

\section{A vacina contra HPV e o câncer cervical}

Conforme a literatura consultada, o câncer cervical tem o HPV como principal agente infeccioso no desenvolvimento das neoplasias intraepiteliais e do processo de carcinogênese. Para a prevenção contra o câncer cervical são utilizados alguns métodos, como a citologia cérvico-vaginal ou colpocitologia oncótica. Trata-se do método mais difundido no mundo para rastreamento das $\mathrm{NICs}^{(15)}$. 
A colpocitologia oncótica é utilizada atualmente como meio de diagnóstico indispensável, devido a vantagens como: baixo custo, fácil realização, tratamento adequado com possibilidade de cura e coleta da amostra realizada por médicos e enfermeiros. Recomenda-se a realização desse exame para mulheres de 25 a 60 anos, com periodicidade anual. Se, após dois exames anuais consecutivos, os resultados mostrarem-se normais, a periodicidade recomendada passa para a cada três anos ${ }^{(15-16-17)}$.

Estudos apontam a vacina contra HPV como método profilático que apresenta eficácia diante do câncer cervical, em virtude de a citologia oncótica possuir algumas limitações, como baixa sensibilidade, baixo valor preditivo positivo e baixa reprodutibilidade ${ }^{(4,10)}$.

Duas empresas farmacêuticas, a Merck Sharp \& Dohme (MSD) e a GlaxoSmithKline (GSK) foram responsáveis pelo desenvolvimento das vacinas. A Gardasil @ ou quadrivalente pertence ao grupo MSD, e a Cervarix ${ }^{\circledR}$ ou bivalente pertence ao $\operatorname{GSK}^{(10,18)}$. A vacina quadrivalente atua contra os tipos $6,11,16$ e 18, e a bivalente, contra os tipos 16 e 18 . Ambas têm mostrado uma redução significativa na incidência de infecções persistentes pelo HPV, apresentando-se seguras, bem toleradas e altamente imunogênicas ${ }^{(3,16-19)}$.
As experiências realizadas em mulheres entre 16 e 26 anos de idade, em um período de 42 meses, comprovaram que a vacina quadrivalente apresenta alta eficácia profilática contra as lesões de baixo grau ou NIC I, assim como no aparecimento de condilomas, sendo essa proteção mantida por até quatro anos ${ }^{(9)}$.

No ano de 2006, com a aprovação da primeira vacina para prevenção de infecção pelo HPV, a Gardasil mostrou-se 100\% efetiva contra os HPVs 16 e 18, responsáveis por $70 \%$ dos cânceres cervicais ${ }^{(3,18)}$. De fato, as evidências apontam que tais vacinas têm a capacidade de prevenção de infecções em adolescentes sem exposição anterior ao vírus, mas, em mulheres já infectadas pelo HPV, não se terão benefícios, pois a vacina é profilática (20-21)

Em estudo recente (10), foi evidenciado que a janela de eficácia da vacina é mais elevada antes do início da atividade sexual, por isso esse processo deve ser estabelecido através de ações educativas e do vínculo construído entre profissionais de saúde, pais e adolescentes.

Pesquisas sinalizam para a inclusão de vacinas polivalentes, que assim possam contemplar cerca de cinco a oito tipos de HPV, o que resultaria na extensão da proteção contra mais de $90 \%$ dos tipos oncogênicos do HPV. Tal percepção pretende alertar pesquisadores no campo 
da saúde pública com vistas ao avanço de estudos nessa perspectiva ${ }^{(22)}$.

No Brasil, as vacinas foram aprovadas pela Agência Nacional de Vigilância Sanitária (ANVISA), com indicação para mulheres de 9 a 26 anos e 10 a 25, respectivamente. Contudo, estudos em nível mundial mostraram-se controversos quanto à eficácia das vacinas para mulheres acima de 26 anos. Isso implica na necessidade de se desenvolverem estratégias educativas e preventivas voltadas à saúde sexual e reprodutiva $^{(13,23-24)}$.

É verdade, que algumas pesquisas com a vacina contra HPV demonstraram eficiência de $100 \%$ para a prevenção de NIC II e III, ou adenocarcinoma in situ (AIS), associados aos HPVs 16 e 18, e também de $100 \%$ para lesões da vulva (NIC II / III) ou da vagina (NIC II / III), associadas com os HPVs 16, 18, 11 e 6. Vale enfatizar que a vacinação não exclui a necessidade de rastreamento citológico, pois não elimina isoladamente o câncer cervical $^{(13,23,25)}$.

Corroborando com o parágrafo anterior, afirma-se que o rastreio citológico de rotina é necessário, visto que as vacinas não protegem contra todos os tipos oncogênicos do HPV. Sendo assim, recomenda-se que as mulheres realizem a citologia oncótica, pois estas permanecem em risco quanto a outros genótipos de alto risco do $\mathrm{HPV}^{26)}$.

As vacinas devem ser administradas em três doses, por via intramuscular, com o esquema 0, 2 e 6 meses (Gardasil $®)$ e e 0,1 e 6 meses (Cervarix (®). Verificou-se a possibilidade de uma quarta dose, pelo fato de os níveis de anticorpos, após a terceira dose, diminuírem progressivamente e atingirem a estabilidade aos 18-24 meses, permanecendo estáveis até 60 meses. A administração de uma quarta dose seria, então, para a indução de aumento da memória imunológica. Todavia ainda não se sabe se o declínio dos níveis de anticorpos de HPV irá exigir uma quarta dose $\mathrm{e}^{(10,20)}$.

Sobre os eventos adversos apresentados, estes foram relacionados à dor no local da administração, com intensidade leve ou moderada, e presença de eritema localizado ${ }^{(10,23)}$.

Quanto ao custo na América do Norte e na Europa, o preço das três doses da vacina quadrivalente é de cerca de US\$ 360 , valor possivelmente não tolerado por países em desenvolvimento. No Brasil, as duas empresas que produzem a vacina estimaram que, para ser custo-efetiva, cada dose teria o valor de $\mathrm{R} \$ 5,00^{(23)}$.

No entanto, novos gastos com uma vacina para o sistema de saúde devem ser definidos após rigorosa avaliação, pois os programas de rastreio e diagnóstico 
precoce do câncer de colo do útero realizados no momento não podem ser eliminados em curto prazo de tempo, ainda que haja inserção das vacinas no sistema público de saúde. Estudos de avaliação de custo e efetividade devem ser bem planejados e executados antes de tal decisão ${ }^{(11,13)}$.

Alguns estudos destacam que menos de $10 \%$ entre as participantes reconheciam o HPV como preditor do câncer cervical, sendo este um viés para aceitação da vacina. $O$ processo de aceitação da vacina apresenta alguns entraves sociais, econômicos, crenças culturais, religiosas, com impacto sobre o comportamento sexual dos adolescentes e o grau de conhecimento sobre a doença e o HPV ${ }^{(10)}$.

$$
\text { Em última análise, as vacinas }
$$
profiláticas são bem toleradas mundialmente, no entanto não estarão disponíveis em curto prazo para a vacinação pública, pois envolvem, principalmente nos países em desenvolvimento restrições econômicas e outras questões. Apesar dos estudos de custo-efetividade da vacina contra HPV se mostrarem uma forma de produção de conhecimento e de utilidade no apoio às decisões dos gestores, deve-se reconhecer que as decisões em si, e sua tradução em iniciativas políticas e técnicas, passam por processos muito mais complexos e abrangentes $^{(27)}$.

\section{CONSIDERAÇÕES FINAIS}

A vacina profilática contra HPV emerge como uma relevante medida de controle tanto de lesões intraepiteliais precursoras quanto da instalação da neoplasia maligna. Todavia, outras medidas profiláticas, como a citologia oncótica, e a prevenção contra as doenças sexualmente transmissíveis não podem ser excluídas. Desse modo, os tipos de intervenções preventivas - primárias e secundárias - devem contribuir para a redução dos índices apontados neste estudo, em que todos os atores sociais, com enfoque para os gestores da saúde, devem ser sensibilizados para a adesão de tais medidas.

Entende-se que os resultados deste estudo representam uma ferramenta capaz de fomentar, nos diferentes atores envolvidos, momentos de sensibilização e reflexão, para prevenir o câncer cervical, principalmente no tocante à profilaxia relacionada ao papilomavírus humano. Faz-se necessário o reforço para as orientações de educação à saúde da população mais vulnerável, reforçando a importância das estratégias que contemplem o processo de imunização e melhoria das políticas públicas de saúde, observando-se como ponto de partida a construção e a reconstrução de saberes. 


\section{REFERÊNCIAS}

1. Instituto Nacional de Câncer. Estimativa 2010: incidência de Câncer no Brasil. Rio de Janeiro: Instituto Nacional de Câncer; 2009.

2. Instituto Nacional de Câncer. Ações de Enfermagem para o controle de câncer: uma proposta de integração ensino-serviço. Rio de Janeiro: Instituto Nacional de Câncer; 2008.

3. Rosa MI, Medeiros LR, Rosa DD, Bozzeti MC, Silva FR, Silva BR, et al. Papilomavírus humano e neoplasia cervical. Cad Saúde Pública 2009; 25: 953 64.

4. Nadal SR, Manzione CR. Vacinas Contra o Papilomavirus Humano. Rev Bras Coloproct 2006; 6: 337-40.

\section{Mendes KDS, Silveira RCCP, Galvão}

CM. Revisão integrativa: método de pesquisa para a incorporação de evidências na saúde e na enfermagem. Texto contexto-enferm. 2008; 17:758-64.

6. Whittemore R, Knafl K. The integrative review: updated methodology. J Adv Nurs 2005; 52: 546-53.

7. Souza MT, Silva MD, Carvalho R. Revisão integrativa: o que é e como fazer. Einstein 2010; 8: 102-6.

8. Rama CH, Villa LL, Pagliusi S, Andreoli MA, Costa MC, Aoki AL, et al. Awareness and knowledge of HPV, cervical cancer, and vaccines in young women after first delivery in São Paulo, Brazil - a cross-sectional study. BMC Womens Health 2010; 10:1-7.

9. FUTURE I/II Study Group, Dillner J, Kjaer SK, Wheeler CM, Sigurdsson K, Iversen OE, Hernandez-Avila M, et al. Four year efficacy of prophylactic human papillomavirus quadrivalent vaccine against low grade cervical, vulvar, and vaginal intraepithelial neoplasia and anogenital warts: randomised controlled trial. BMJ 2010; 341.

10. Lepique AP, Rabachini T, Villa LL. HPV vaccination: the beginning of the end of cervical cancer? - A Review. Mem Inst Oswaldo Cruz 2009; 104: 1-10.

11. Pirhardt CR, Mercês NNA. Fatores de risco para Câncer de mama: Nível de conhecimento dos acadêmicos de uma universidade. Rev enferm UERJ 2009; 17: 102-6.

12. Moura ADA, Silva SMG, Farias LM, Feitoza AR. Conhecimento e motivações das mulheres acerca do exame de Papanicolau: subsídios para a prática de enfermagem. Rev RENE 2010; 11: 94-104.

13. Kohli M, Ferko N, Martin A, Franco EL, Jenkins D, Gallivan S, et al. Estimating the long-term impact of a prophylactic human papillomavirus 16/18 vaccine on the burden of cervical cancer in the UK. Br J Cancer 2007; 96: 143-50.

14. Giraldo PC, Silva MJPMA, Fedrizzi EN, Gonçalves AKS, Amaral RLG, Eleutério Jr J, et al. Prevenção da infecção por HPV e lesões associadas com o uso de vacinas. DST $\mathrm{j}$ bras doenças sex transm 2008; 20: 132-40.

15. Rocha GA, Melo VH. Biologia molecular no rastreamento das neoplasias cervicais uterinas. Femina 2010; 38: 16772 .

16. Murta EFC. Vacina contra o HPV ou contra o câncer de colo uterino? Rev Bras Ginecol Obstet 2007; 29: 548.

17. Villa MCE, Pereira WR. As políticas públicas e a atenção ao câncer do colo do útero no Estado de Mato Grosso - uma abordagem crítica. Rev. Eletr. Enf. [Internet]. 2009; 11(4): 1037-42. Available from: 
http://www.fen.ufg.br/revista/v11/n4/pdf/v 11n4a31.pdf.

18. Instituto Nacional de Câncer. Plano de ação para redução da incidência e mortalidade por câncer do colo do útero: sumário executivo. Rio de Janeiro: Instituto Nacional de Câncer; 2010.

19. Parry J. Vaccinating against cervical cancer. Bull World Health Organ 2007; 85:89-90.

20. Muñoz N, Reina JC, Sánchez GI. La vacuna contra el virus del papiloma humano: una gran arma para la prevención primaria del cáncer de cuello uterino. Colomb Med 2008; 39:196-204.

21. Wolschick NM, Consolaro MEL, Suzuki LE, Boer CG. Câncer do colo do útero: tecnologias emergentes no diagnóstico, tratamento e prevenção da doença. Rev bras anál clin 2007; 39: 123-9.

22. Bosch FX, Castellsagué X, Sanjosé S. HPV and cervical cancer: screening or vaccination?. Br J Cancer 2008; 98:15-21.

23. Feliciano C, Christen K, Velho MB. Câncer de colo uterino: realização do exame Colpocitológico e mecanismos que ampliam sua adesão. Rev enferm UERJ. 2010; 18:75-9.

24. Lopes HV. Sobre a vacina contra o HPV. Rev panam infectol 2006; 8: 50-1.

25. Nadal LRM, Nadal SR. Indicações da vacina contra o papilomavírus humano. Rev Bras Coloproct 2008; 28: 124-6.

26. Dawar M; Deeks S; Dobson S. Human papillomavirus vaccines launch a new era in cervical cancer prevention. CMAJ 2007; 177:456-61.

27. Novaes HMD. A vacina contra HPV e o câncer de colo de útero: desafios para a sua incorporação em sistemas de saúde. Rev Bras Epidemiol 2008; 11: 505-25. 